\title{
Être ou ne pas être un ancien soldat de l'Empire?
}

Un processus identitaire dans les campagnes de Haute-Bretagne au xixe siècle

To be or not to be an Empire veteran? Identity processes in the countryside in 19th century

\section{Mathieu Marly}

\section{(2) OpenEdition}

\section{Journals}

\section{Édition électronique}

URL : http://journals.openedition.org/abpo/2008

DOI : $10.4000 /$ abpo.2008

ISSN : 2108-6443

\section{Éditeur}

Presses universitaires de Rennes

Édition imprimée

Date de publication : 30 mai 2011

Pagination : 103-116

ISBN : 978-2-7535-1691-5

ISSN : 0399-0826

\section{Référence électronique}

Mathieu Marly, "Être ou ne pas être un ancien soldat de l'Empire? ", Annales de Bretagne et des Pays de l'Ouest [En ligne], 118-2 | 2011, mis en ligne le 30 juin 2013, consulté le 21 avril 2019. URL : http:// journals.openedition.org/abpo/2008; DOI : 10.4000/abpo.2008 


\title{
Être ou ne pas être un ancien soldat de l'Empire?
}

\section{Un processus identitaire dans les campagnes de Haute-Bretagne au XIX ${ }^{\mathrm{e}}$ siècle}

\author{
Mathieu MARLY \\ Agrégé d'Histoire \\ doctorant IRHIS-Université Lille 3
}

Le mercredi 18 août 1858, une charrette transportant quelques vieillards quittait Redon pour se rendre aux festivités données à Rennes en présence de Napoléon III. Ces vieillards portaient la médaille de SainteHélène ${ }^{1}$. Un ancien officier des armées impériales dirige alors ce détachement en qualité de " commandant militaire ". À ses ordres se trouvent un ancien soldat " commissaire aux vivres ", deux vieux " porte-drapeaux " et un " tambour". La consigne de marche a été minutieusement établie. Ces hommes doivent répondre aux exigences d'un règlement qui les replonge dans la rigueur quelque peu artificielle ${ }^{2}$ des longues marches de la Grande Armée :

"Nul ne pourra s'écarter du convoi sans autorisation du chef, sous quelque prétexte que ce soit et ne pourra aller dans une auberge autre que celle où se rendra le détachement.

Toute infraction à la consigne ou aux ordres du chef sera [suivie] de la privation de la prochaine ration de boisson.

Le retard d'un homme ne pourra en aucun cas empêcher le convoi de continuer sa route."

1. Créée par le décret du 12 août 1857, cette médaille est destinée à tous les anciens soldats ayant combattu de 1792 à 1815. Les anciens soldats de la République sont alors largement minoritaires.

2. La minutie du règlement reflète davantage cette « fonctionnarisation de l'esprit militaire " et ce dogme de l'obéissance absolue qui naissent dans l'Armée Française à partir de 1818, qu'elle ne renvoie à l'état d'esprit des armées napoléoniennes. Voir Raoul GiRARDET, La Société militaire de 1815 à nos jours, Paris, Perrin, 2001, 338 p., première édition 1953. 
Deux jours plus tard, arrivés à Rennes, les vieux médaillés de Redon doivent suivre un itinéraire prévu par la consigne :

"En passant devant les postes militaires, au Polygone, à l'Arsenal, à l'Ecole militaire, à la Mairie, les médaillés crieront une fois : Vive l'Empereur! Si le poste ou la sentinelle ne rend pas les honneurs militaires, le convoi continuera sa route, s'il rend les honneurs militaires, le convoi s'arrêtera, le tambour donnera un ban et les médaillés crieront trois fois : Vive l'Empereur! En passant devant le calvaire et devant la cathédrale, le convoi s'arrêtera et les drapeaux feront trois saluts $[\ldots]^{3}$."

Puis le convoi de Redon rejoint les autres médaillés à cette dernière revue qui, assure alors L'Auxiliaire Breton, devait remplir d'une juste émotion les spectateurs groupés autour du Champ de Mars :

" [Les gendarmes] étaient suivis des vieux médaillés de Sainte Hélène, portant le buste de Napoléon $1^{\mathrm{er}}$, couronné de lauriers; dans leurs rangs, on apercevait encore quelques débris des uniformes de la Grande Armée. À ce moment l'enthousiasme était à son comble, et bien des larmes coulaient; un vieillard s'est presque agenouillé en passant devant leurs Majestés Impériales ${ }^{4}$."

Aussi émouvant soit-il, ce spectacle ne laisse pas d'intriguer. À suivre l'enthousiasme des médaillés de Sainte-Hélène, c'est une figure bien connue des lecteurs de Balzac qui vient à l'esprit : celle du "vieux débris de la Grande armée ", ancien grognard vivant du souvenir de la gloire impériale. Les spectateurs du Champ de Mars n'ignoraient probablement pas cette figure majeure de l'imaginaire national qui s'impose alors de manière récurrente dans la littérature, les chansons et les discours politiques ${ }^{5}$.

Or, ce topos est au cœur de l'entreprise de légitimation du pouvoir impérial à l'œuvre dans l'institution des médailles de Sainte-Hélène ${ }^{6}$. Il ressort des discours des autorités, tel celui du préfet d'Ille-et-Vilaine qui lors des remises publiques de la médaille de Sainte-Hélène, quelques semaines plus tôt, comparait déjà les médaillés aux " compagnons du héros des Temps modernes $^{7}$ ". De plus, leur présence au sein des couches populaires bretonnes devait contribuer au succès politique du voyage de Napoléon III en entraînant tout au long du parcours l'enthousiasme de leurs concitoyens ${ }^{8}$.

3. Arch. dép. d'Ille-et-Vilaine, 3 Z 49 : consigne pour les médaillés de Sainte-Hélène (10 août 1858).

4. L'Auxiliaire Breton (samedi 21 août 1858).

5. Natalie Petrteau, Napoléon de la mythologie à l'histoire, Paris, Seuil, Points Histoire, 2004, 458 p., première édition 1999. Sudhir HAZAREESINGH, La Légende de Napoléon, Paris, Tallandier, 2005, 414 p., première édition 2004.

6. Sudhir HAZAREESINGH, "La légende napoléonienne sous le second Empire : les médaillés de Sainte-Hélène et la fête du 15 août ", Revue Historique, n 627 , juillet 2003, p. 564.

7. Journal de Rennes (10 mars 1858).

8. Jean-Marc Roussel, Le Pouvoir en représentation, le voyage officiel de Napoléon III en Bretagne (août 1858), mémoire de maîtrise, sous la direction de Jean-François TANGUY, Rennes 2, septembre 2002, p. 121. 
Rien d'étonnant donc à voir les représentants du régime impérial - journaux " officiels " compris - travestir ces vieillards en thuriféraires nostalgiques du premier Empire.

Cette figure du "vieux de la vieille " pourrait donc sembler suspecte si elle n'était partagée par les anciens soldats eux-mêmes. En effet, les demandes de secours envoyées sous le second Empire abondent en formules stéréotypées et autres professions de foi impériale. En 1850, Louis Coquelin alors cultivateur à Louvigné-du-désert se présente comme " un vieux débris de la Grande Armée " quand François Poirier, menuisier à Vitré, commence ainsi sa lettre adressée au ministre de la Guerre : «Encore un soldat de l'Empire, débris de l'ancienne armée, blessé au champ de bataille! » Quant à Mathurin Denis, employé aux forges de Paimpont, il assure " ses sentiments de dévouement et d'admiration pour l'héritier de l'empereur sous lequel il a combattu ${ }^{9}$ ".

Mais la signification que recouvrent ces déclarations est loin d'être évidente. À se limiter aux fortes démonstrations d'enthousiasme des médaillés et aux comptes rendus des cérémonies impériales, on ne peut comprendre ce qui sous-tend l'adhésion de ces vieillards au modèle du "vieux de la vieille " qui leur est alors proposé.

\section{L'envers de la médaille de Sainte-Hélène}

Il faut revenir aux premiers mois de la présidence de Louis-Napoléon Bonaparte pour voir s'esquisser le rapport ambigu reliant progressivement les anciens soldats au nouveau pouvoir. Dès 1849, une circulaire adressée aux préfets invite ces derniers à faire recenser dans toutes les communes les anciens militaires du premier Empire « qui auraient été privés de récompense due à leurs services ${ }^{10}$ ". Cet acte de générosité impériale se précisera dans les années qui suivent, invitant les autorités à recevoir en priorité les demandes d'anciens soldats dont les états de service apparaissent authentiques et ceux dont l'indigence est reconnue. Loin de consacrer un statut d'ancien combattant - en attribuant à chacun une somme fixe d'un montant déterminé par la loi - le système de secours mis en place par le régime s'inscrit dans une logique de charité intégrée au fonctionnement de l'État ${ }^{11}$.

Dès lors, l'insuffisance des ressources budgétaires face au nombre de demandes et la difficulté des anciens soldats à produire les preuves de leurs états de service inscrivent chaque réclamant dans une logique concurrentielle face à l'administration. Bien vite, ce ne sont plus les états de service ni l'indigence qui assurent l'obtention d'un soutien matériel, mais la capacité

9. Arch. dép. d'llle-et-Vilaine, 2 R 264 : lettre de Coquelin au Président de la République (26 juin 1850), lettre de Poirier au ministère de la Guerre (8 juin 1850); 2 R 357 : lettre de Mathurin Denis au ministre de la Guerre (25 août 1854).

10. Arch. dép. d'Ille-et-Vilaine, circulaire du ministère de l'intérieur (6 décembre 1849)

11. Jean-Luc MARAIS, Histoire du don en France de 1800 à 1939. Dons et legs charitables, pieux et philanthropiques, Rennes, PUR, 1999, p. 44. 
à faire entendre sa qualité d'ancien soldat de l'Empire, ce qui soumet ces vieillards à une véritable injonction identitaire et mémorielle ${ }^{12}$. Les traces d'anciennes blessures, les récits de bataille et les professions de foi impériales sont autant de passeports pour l'obtention d'un secours. Ce système profite alors aux plus audacieux qui savent susciter les recommandations de personnages hauts placés et mettre en scène leur passé militaire dans les armées impériales.

Par son habileté à faire valoir ses droits, l'ancien soldat Pierre Vachot Desmirail, illustre cette dérive. Dès la mise en place des secours, ce dernier obtient les recommandations du préfet à travers une correspondance suivie et larmoyante ${ }^{13}$. L'absence de preuves sérieuses concernant son indigence - une enquête préfectorale menée en 1850 semble plutôt prouver le contraire - ne l'empêche pas d'obtenir dans les années qui suivent plusieurs secours ponctuels, une pension viagère et la somme de 1635 francs au titre d'ancien soldat de l'île d'Elbe ${ }^{14}$, soit bien plus que ne pouvaient espérer d'anciens soldats pauvres et sans soutiens. Le jeudi 19 août 1858, cet ancien soldat apparaît au détour du chemin qui conduit le couple impérial de Saint-Malo à Rennes, sous un arc de triomphe qu'il a lui-même confectionné et qui porte l'inscription suivante : «À l'Empereur. À l'Impératrice. À leur fils bien aimé, nous jurons fidélité : un vieux soldat de l'Île d'Elbe à sa Majesté Napoléon III. "Quelques jours plus tard, L'Union Malouine révèle que l'empereur a paru touché par l'œuvre du vétéran et lui a " pressé la main ". Contrarié par la sobriété de cet article, Vachot Desmirail écrit à la Chronique de Fougères pour fournir plus de détails sur cette rencontre, précisant les derniers mots de l'empereur : "Mon ami, je ne vous oublierai pas ${ }^{15}$. "Si elle n'est peut être pas exempte d'une réelle ferveur, une telle publicité pouvait aller dans le sens de nouvelles recommandations...

Pour illustrer avec excès ce type de comportement, cet ancien soldat ne constitue pas un cas isolé. Plusieurs vétérans se sont trouvés sur le chemin de Napoléon III et ont obtenu des secours ponctuels ou des pensions viagères ${ }^{16}$. Du reste, pour beaucoup, encore tenus à l'écart de la générosité impériale en 1858, l'inscription sur les registres des médaillés de Sainte-Hélène, si elle ne donne droit à aucune pension, pouvait être

12. Cet aspect de la générosité impériale n'a pas échappé à quelques contemporains dont Auguste Pointeau du Ronceray, notable rennais et futur médaillé de Sainte-Hélène, qui le déplore dans sa correspondance : "Voilà hélas le cas que l'on fait de justes réclamations réclamées à grand bruit, à son de trompette et de grosse caisse, à de pauvres vieux soldats endormis ", Arch. dép. d'Ille-et-Vilaine, 1 J 208 (lettre à son fils, 13 juillet 1856).

13. Cet ancien soldat va jusqu'à menacer de se suicider pour obtenir des secours! Arch. dép. d'Ille-et-Vilaine, 2 R 297 (lettre de Vachot Desmirail au préfet, 15 novembre 1850).

14. Arch. dép. d'Ille-et-Vilaine, 2 R 297 : enquête préfectorale sur Pierre Vachot Desmirail (1850).

15. L'Union Malouine et dinannaise (dimanche 22 août 1858); Chronique de Fougères (samedi 24 août 1858).

16. Pour faire face à la prodigalité de l'empereur, la Grande Chancellerie demande alors au préfet une enquête sur chaque bénéficiaire. Arch. dép. d'Ille-et-Vilaine, la Grande Chancellerie de la Légion d'Honneur au préfet (4 octobre 1858). 
perçue comme un passage obligé vers la reconnaissance des autorités ${ }^{17}$. Derrière l'enthousiasme des vieux médaillés, si souvent relevé dans les études portant sur la médaille de Sainte-Hélène, c'est un échange de bons procédés qui est à l'œuvre entre ces anciens soldats et le pouvoir impérial. Celui-ci ne relève ni d'une froide manipulation des autorités ni d'un calcul toujours intéressé des anciens soldats, mais s'insère dans un mécanisme de secours qui pousse ces hommes revenus à la vie civile à adopter les codes du vieux troupier bonapartiste en échange de distinctions honorifiques et de secours pécuniaires.

La médaille de Sainte-Hélène uniformise donc ces parcours en apposant le sceau du nouveau régime impérial sur des processus identitaires autrement plus complexes. Contre cette représentation envahissante du vieux grognard bonapartiste, Natalie Petiteau a montré la complexité et la diversité de ces destins ${ }^{18}$. Loin de ressembler uniformément aux vieux soldats de la légende que semblent incarner les médaillés de Sainte-Hélène, ce groupe se caractérise avant tout par son hétérogénéité. Il ne forme pas dans la société française du XIX siècle un " groupe social " aux contours bien définis; l'expérience commune dans les armées napoléoniennes n'étant pas socialement ni politiquement structurante après 1815 .

Les conclusions de Natalie Petiteau invitent donc à se pencher sur les processus identitaires à l'œuvre chez ces anciens soldats avant que la médaille de Sainte-Hélène ne les fige dans la posture du " vieux débris de la Grande Armée ». Le concept de " groupe social " n'étant pas ici pertinent, ce sont des constructions identitaires singulières conçues comme des lieux d'interférences entre des logiques individuelles, sociales, culturelles mais également politiques qui doivent être prises en compte pour comprendre comment ces hommes se sont appropriés ou pas l'identité d'ancien soldat de Napoléon après 1815. À travers ce questionnement, c'est aussi l'espace de réinsertion des anciens soldats qui est étudié, leur expérience guerrière prenant son sens dans le dialogue établi avec la société civile avant l'institution de la médaille de Sainte-Hélène en 1858. Les représentations de l'armée, de la légende napoléonienne et de l'horizon national dans les campagnes de Haute Bretagne apparaissent ainsi en creux à travers l'étude de ce groupe.

\section{Les vétérans au village}

L'enquête menée en Ille-et-Vilaine appuie les conclusions de Natalie Petiteau ${ }^{19}$. Les vétérans de Napoléon ne forment pas dans ce département

17. La remise des médailles de Sainte-Hélène ne clôt pas l'aide matérielle de l'État. Deux recensements sont à nouveau effectués en 1860 puis en 1869 pour venir en aide aux anciens soldats.

18. Natalie Petiteau, Lendemains d'Empire. Les anciens soldats de Napoléon dans la France du XIXe siècle, Paris, La Boutique de l'Histoire, 2003, 396 p.

19. Mathieu MARLY, Les Vétérans de la Grande Armée dans le département d'Ille-et-Vilaine au XIX siècle, mémoire de Master 2 sous la direction de Patrick Harismendy, Rennes 2, 2006, 257 p. Pour étudier la réinsertion de ce groupe après 1815, un corpus de 3745 vété- 
un groupe social cohérent. Mais si l'inventaire des conditions sociales révèle une très grande hétérogénéité de position et de fortune, l'éventail des conditions de réinsertion tend à se resserrer autour de quelques parcours plus représentatifs.

C'est avant tout dans les campagnes que s'effectue la plus grande part des retours à la vie civile : $72 \%$ des vétérans recensés sous le second Empire sont alors domiciliés dans les communes rurales du département. Les mentions professionnelles contenues dans les demandes de secours témoignent d'une majorité de réinsertions dans les métiers de la terre, soit plus de $50 \%$ des 464 mentions professionnelles auxquelles on peut ajouter, dans un contexte de forte pluriactivité, une grande partie des professions artisanales déclarées ${ }^{20}$. La réinsertion dans les collectivités villageoises, pour n'être pas exclusive, s'impose alors comme l'expérience la plus commune. Elle se distingue des solidarités de groupe formées dans quelques institutions spécialisées, comme les Invalides, car elle confronte l'identité et la mémoire de ces hommes aux seuls repères et aux seules attentes des collectivités villageoises. En l'absence de reconnaissance de l'État, d'associations d'anciens combattants ou de liens de sociabilité clairement identifiés, c'est dans le dialogue des anciens soldats avec les populations rurales que peuvent être cernées les étapes de ces processus identitaires ${ }^{21}$.

À contre-courant des représentations littéraires, les données recueillies à l'échelle communale peuvent surprendre : toutes soulignent la profonde intégration de ces hommes aux collectivités villageoises. Loin d'être ces êtres " sans feu ni lieu " rendus hostiles au mariage par des années de campagnes militaires, les vétérans des communes de Gahard, Gévezé et Princé illustrent par leurs parcours un véritable désir d'enracinement familial et villageois. Sur 34 anciens soldats, plus de la moitié se sont mariés dans leurs communes de naissance et ont eu au moins quatre enfants ${ }^{22}$. De plus, il est possible de déceler parmi eux les signes d'un profond attache-

rans a été constitué à partir des demandes de secours et des registres de la médaille de Sainte-Hélène sous le second Empire. Les informations fournies par le corpus ont été croisées avec les méthodes d'une histoire " au ras du sol " effectuée dans trois villages du département. Enfin les sources les plus diverses (journaux, correspondances privées, etc.) ont été mises à contribution.

20. Natalie Petiteau a obtenu un pourcentage comparable concernant les 561 mentions professionnelles collectées dans le Vaucluse. Natalie PetrTEAU, Lendemains d'empire..., op. cit., p. 228.

21. Les solidarités de groupe en milieu urbain ont été étudiées par Natalie Petiteau à travers les Invalides et sa succursale à Avignon. La recherche des réseaux d'interconnaissance dans les trois communes aboutit aux même conclusions que celle observées par Natalie Petiteau dans la commune d'Ansouis : il est difficile de discerner dans les quelques liens observés la part de la sociabilité traditionnelle d'une solidarité liée à l'expérience militaire. Natalie PetrTeAu, Lendemains d'empire..., op. cit., p. 314. Les associations d'anciens combattants de l'Empire, présentes dans d'autres régions, sont à notre connaissance, inexistantes dans ce département avant l'institution de la médaille de Sainte-Hélène.

22. Ces informations sont extraites des états civils des communes de Gahard, Gévezé et Princé conservées aux Archives départementales de l'Ille-et-Vilaine. 
ment à cette " petite patrie " que constitue la paroisse dans les campagnes de Haute-Bretagne. Ainsi, lorsqu'en 1821 les habitants de la commune de Gahard décident de vendre des portions de terrains privés pour acheter une deuxième cloche, sacrifiant ainsi à cet " esprit de clocher " décrit par Alain Corbin; deux anciens soldats se présentent à la mairie pour sacrifier respectivement " un bout de terrain dans les Landes " et une " pâture ${ }^{23}$ ". L'inscription d'anciens soldats de la commune de Gévezé sur les registres de la Fabrique et de la confrérie du "Saint-Scapulaire " établie en 1826 souligne également leur implication dans cette sociabilité religieuse caractéristique de la paysannerie bretonne. Enfin, si l'analyse menée « au ras du sol " dans ces trois communes souligne la présence de quelques vétérans tenus à l'écart de la sociabilité villageoise ${ }^{24}$, ces derniers le doivent plus souvent à leur statut de " horsain " qu'à la singularité de leur expérience militaire.

Un grand nombre de vétérans évolue donc dans ce relatif " entre-soi " des collectivités villageoises. Néanmoins, l'expérience unique qui est la leur laisse à penser qu'ils disposent vis à vis de leurs concitoyens d'un crédit suffisant pour assurer des fonctions importantes à l'échelle locale. L'ancien soldat devenu maire de son village, figure présente dans les représentations du temps ${ }^{25}$, pose la question de la notabilité des vétérans impériaux.

À la tête du pouvoir municipal de Renac à partir de 1848 - quelques semaines avant l'élection de Louis-Napoléon Bonaparte - Joseph Delanoë semble correspondre au modèle du vieux grognard bonapartiste. Le souspréfet de Redon décrit ainsi le maire de Renac en 1854 : «Ancien grenadier de la Garde impériale, [le maire] a hélas le défaut d'être ivre à l'état normal; mais en revanche la qualité d'aimer tous les souvenirs de l'empire. " Dans ses états de service, la description précise de la bataille de Waterloo laisse entendre le prestige tiré de son passage dans les armées impériales. Mais il est possible que cet ancien soldat tienne sa notabilité d'une fortune et d'une forte assise familiale qui lui permet d'être nommé adjoint au maire dès $1830^{26}$. Dans bien des cas, en effet, l'élection des vétérans à la tête de leurs communes consacre une notabilité acquise par une solide position sociale et foncière.

23. Arch. dép. d'Ille-et-Vilaine, E Dépôt administratif de Gahard, art. 2, procès verbal (12 octobre et 15 octobre 1821).

24. Ces écarts sont mesurés à l'aide d'un faisceau d'indices : lieu de naissance, participation aux institutions villageoises, emploi, nom des enfants, etc.

25. David M. HopkIN, Soldier and Peasant in French Popular Culture 1766-1870, Woobridge and Rochester, The Boydell press for the Royal Historical Society, 2003, p. 294-297. Cette représentation s'appuie sur une réalité tangible en Lorraine. Voir à ce sujet Michel SALVIAC, "Les maires anciens soldats : l'arrondissement de Commercy " dans Maurice AgulHoN, dir., Les Maires en France du Consulat à nos jours, Paris, Publications de la Sorbonne, 1986, p. 329-344.

26. Arch. dép. d'Ille-et-Vilaine, 2 R 357 - États de service de Joseph Delanoë (1850), 1 M 186 : le sous-préfet de Redon au préfet (16 août 1854), E Dépôt administratif de Renac. 
Il en va de même si l'on considère l'élection des conseillers municipaux élus à partir de 1831. À l'échelle des trois communes sélectionnées, seuls deux anciens soldats participent aux conseils municipaux. Mais leur élection par les administrés les plus imposés de chaque commune doit beaucoup à leur qualité de propriétaires aisés. À l'inverse, Jean Delabarre, laboureur moyen qui compte pourtant dix ans de service, tente sans succès d'intégrer le conseil municipal de Gévezé lors des élections du 27 janvier 1833, prouvant par là que l'expérience militaire n'assure pas une notabilité équivalente à la richesse foncière dans cette commune ${ }^{27}$. En définitive, on distingue mal à travers la notabilité de quelques vétérans le prestige proprement militaire d'une notabilité obtenue par des voies plus traditionnelles.

La participation massive des anciens soldats à la formation des Gardes nationales dans les premières années de la monarchie de Juillet apparaît $a$ contrario comme un signe tangible de valorisation de l'expérience militaire. Beaucoup reçoivent alors de modestes galons au sein de ces humbles - et souvent éphémères - milices rurales. À Gahard, deux vétérans sont élus à ces postes quand, dans la commune de Gévezé, la quasi-totalité des sousofficiers et officiers élus en 1833 sont d'anciens militaires impériaux. Lors de la formation de ces unités, certains vétérans occupent alors la position d'instructeur et prouvent ainsi que leur expérience des armes est reconnue par leurs concitoyens. Pour autant, ces élections confirment davantage la reconnaissance d'un domaine d'expertise que le prestige social des intéressés. Pierre Crespel et Pierre Guézille, tous deux vétérans et notables reconnus dans leurs communes respectives obtiennent ainsi le grade de sous-officier quand ce sont des vétérans plus obscurs qui accèdent aux grades de capitaines. À Gévezé, les dix années d'expérience militaire de Jean Delabarre, insuffisantes pour le faire entrer au conseil municipal, lui permettent cette fois d'occuper le grade le plus élevé dans la Garde nationale ${ }^{28}$. Mais si l'élection au grade d'officier valide dans ce cas les années d'expérience militaire, elle ne présente pas pour autant d'enjeu politique majeur à l'échelle communale ${ }^{29}$. De plus, ces petites unités, surtout en milieu rural, disparaissent dans les années qui suivent, ce qui prive ces anciens soldats d'une forme de reconnaissance instituée au sein des collectivités villageoises ${ }^{30}$.

En définitive, exceptées les fonctions exercées dans la Garde nationale, la qualité d'ancien militaire impérial s'avère rarement suffisante pour assu-

27. Arch. dép. d'Ille-et-Vilaine, E dépôt administratif de Gahard (art. 2 et art. 9) et Gévezé (art.17).

28. Arch. dép. d'Ille-et-Vilaine, E dépôt administratif de Gahard (art. 37) et Gévezé (art. 55).

29. Voir Yann Lagadec, Pouvoir et politique en Haute-Bretagne rurale. L'exemple de Louvigné-de-Bais (XVI'-XIX siècle), doctorat sous la direction d'Alain Croix, Rennes 2, 2003, p. 685 .

30. Erwan Соват, La Garde nationale en Ille-et-Vilaine des Cents Jours à la monarchie de Juillet (1815-1834), mémoire de maîtrise sous la direction de Roger Dupuy, Rennes 2, 1992 , p. 67. 
rer une notabilité reconnue par les différentes institutions villageoises. Ce prestige proprement militaire apparaît discrètement, particulièrement à partir de 1848, assurant un surcroît de notabilité aux notables et une place mineure dans les institutions à quelques vétérans qui ont su faire valoir leur expérience militaire. Cependant, ces hommes peuvent utiliser d'autres moyens pour être " reconnus" par leurs contemporains.

\section{À la recherche des signes identitaires}

Sous le second Empire, certaines demandes de secours portent les signes du prestige informel de quelques anciens soldats. Dans la commune de La Chapelle-du-Lou, le maire témoigne ainsi pour Jean Lechaux « qu'il est de notoriété publique qu'il a servi sous l'empire ». À Montfort, Pierre Roulin fait figure de célébrité, cet ancien hussard ayant selon les mots du maire " toujours proclamé la valeur de Napoléon ${ }^{\text {er }}$, ce qui lui a valu le surnom de Guerrier ${ }^{31}$ ". Mais ces quelques exemples restent d'un intérêt limité. Outre qu'ils s'inscrivent dans l'échange de bons procédés à l'œuvre dans le mécanisme des secours, ils n'expliquent pas pourquoi certains soldats ont décidé de mettre en avant leur passage dans les armées napoléoniennes. Ces "stratégies identitaires " peuvent être suivies " au ras du sol ", dans les archives produites par les communes rurales ${ }^{32}$.

À Gahard, Gevezé et Princé, les vétérans se définissent avant tout par leur profession ou leur qualité de propriétaire. Les actes de décès mentionnent exceptionnellement leur qualité d'anciens soldats, soulignant par là que l'entourage familial et le voisinage ne considèrent pas leur passé militaire comme un trait majeur de leur identité. De manière significative, les anciens soldats qui s'affirment comme tels se distinguent par leur indigence au sein de ce groupe. À Gévezé, Ambroise Duval et Pierre Rouyer signalent leurs qualités d'anciens soldats de l'Empire dans le recensement de 1861 et dans leurs actes de décès. Ces deux hommes à l'expérience militaire significative, l'un en Russie, l'autre en Espagne figurent parmi les administrés les plus pauvres de la commune. Le constat est identique à Gahard où les seules mentions de ce passé militaire concernent deux vétérans recensés sur la liste des indigents à secourir établie en 1854. L'expérience militaire semble alors pour ces hommes un moyen de se signaler au moins à la charité d'autrui, au plus à la reconnaissance de leurs concitoyens. Les vétérans pauvres ou marginaux seraient ainsi plus à même de faire valoir leur identité d'ancien soldat de l'Empire au moment même où celle-ci, par le biais de la médaille de Sainte-Hélène, leur confère une aura de respectabilité.

31. Arch. dép. d'Ille-et-Vilaine, 2 R 297 : le maire de la Chapelle du Lou au préfet (9 avril 1850); 2 R 263 : le maire de Montfort au préfet (12 novembre 1858).

32. Notamment à travers la lecture des états civils, des dénombrements, des listes électorales et des registres cadastraux. Les informations qui suivent sont issues des dépôts administratifs de Gahard et Gévezé dans la série E des Archives départementales de l'Ille-et-Vilaine. 
Reste que ces revendications identitaires sont bien rares avant la reconnaissance officielle de l'État sous le second Empire. Exception notable, le cas de Jean Couapel dans la commune de Gévezé permet d'observer " au microscope " ces processus identitaires. Reconnu selon les mots du maire en 1856 comme " un des meilleurs serviteurs de l'empire ", cet homme pauvre qui exerce le métier de tisserand et reste à l'écart des institutions villageoises est le seul à revendiquer dès 1845 son titre de "militaire en retraite " qu'il continue de porter sur les recensements de 1856 et ce jusque sur son acte de décès le 12 juin $1857^{33}$. Au risque de confronter l'enquête aux aléas des " études pinagotiques ", plusieurs éléments de la vie de Jean Couapel offrent matière à réflexion. Cet ancien soldat est mis en cause par la police au début des années 1850 pour avoir obtenu du maire des bons de poudre alors qu'il ne possédait pas de permis de chasse. Il est également soupçonné par la préfecture d'avoir, pour toucher une pension trimestrielle, recueilli une enfant de l'hospice de Rennes qui ne serait autre que le fruit d'une liaison avec sa propre fille ${ }^{34}$. Le comportement atypique de cet homme et la position modeste qu'il occupe dans la commune pourraient expliquer sa propension à affirmer son identité d'ancien soldat impérial, propension partiellement indéchiffrable et qui reste attachée à la grande singularité de ces parcours d'après guerre.

Si l'analyse vient buter tôt ou tard sur les motivations profondes de quelques individus, force est de reconnaître que ces revendications identitaires ne sont pas monnaie courante avant les années 1850. Dans les campagnes du département, il est probable que les anciens soldats impériaux - ou au moins une grande partie d'entre eux - ne reconnaissent pas l'expérience militaire comme un trait déterminant de leur identité, avant que ne leur soit remise la médaille de Sainte-Hélène. L'erreur consiste alors à ne retenir que les portraits " haut en couleur " réalisés par leurs contemporains, lesquels séduits par la légende impériale se sont intéressés à ces quelques célébrités locales incarnant à leurs yeux toutes les vertus du "vieux de la vieille ${ }^{35}$ ".

Toutefois, une analyse classique fondée sur les quelques traces laissées par ces anonymes dans les archives villageoises reste évidemment limitée. Elles n'épuisent en rien la recherche des nombreuses voies de remémoration par lesquelles les anciens soldats témoignent de leur expérience militaire. La parole du vétéran de l'Empire, si souvent caricaturée dans l'imagerie napoléonienne et la littérature du temps, reste pourtant un des principaux vecteurs d'identification. Hors de toute reconnaissance offi-

33. Arch. dép. d'Ille-et-Vilaine, 2 R 264 : demande de secours de Jean Couapel (1856), E dépôt administratif de Gévezé : art. 28/art. 49; Etat civil de Gévezé.

34. Arch. dép. d'Ille-et-Vilaine, E dépôt administratif de Gévezé : art. 21 : le préfet au maire (23 octobre 1852); art. 92 : le préfet au maire (11 octobre 1852) et (7 juillet 1855).

35. La description du brigadier Leclere, surnommé " le dragon " dans les " Cahiers de vieux soldats de la Révolution et de l'Empire " est un modèle du genre. Voir, Jean CHATTON et Nicolas LECLERE, Cahiers de vieux soldats de la Révolution et de l'Empire, publiés et annotés par M. E. Gridel et le capitaine Richard, Paris, Chapelot, 1903, p. 111-117. 
cielle, d'association commémorative et de liens de sociabilité clairement établis entre ces vétérans, cette parole s'ordonne, à moins d'un interminable radotage, sur les attentes de l'entourage et les références de l'auditoire. Elle se dispense alors dans l'espace domestique, les cabarets, les veillées campagnardes alors fréquentes ${ }^{36}$ ou lors des repas de noces. Le cas des vétérans appelés à remplir le rôle de témoin dans les mariages pose la question de cette fonction d'amuseur et de conteur lors de ces banquets. Si dans la commune de Princé, Louis Bonnel et Jean Lambarré doivent probablement leurs fonctions répétées de témoins à leur notabilité ou à leur métier de tailleur responsable du trousseau de la mariée; à Gahard la présence de Jacques Heuzé à 35 mariages entre 1837 et 1848 semble plus curieuse. Il n'est pas impossible que cet ancien soldat, qui indique dans ses états de service avoir reçu son congé à Fontainebleau en 1814, ait pu trouver un auditoire attentif à l'occasion de ces noces ${ }^{37}$.

Par ailleurs, ce rôle de conteur tenu par les anciens soldats est attesté dans la " littérature orale " de la paysannerie du XIX ${ }^{\mathrm{e}}$ siècle $^{38}$. Leur parole est alors intégrée à la culture orale des sociétés rurales auxquelles ils appartiennent. Elle doit séduire et fasciner si elle veut conquérir un auditoire friand de contes et peu regardant sur la vraisemblance des détails de l'expérience militaire. L'essence même des récits réside donc dans cette adaptation à la culture orale et aux attentes de la collectivité villageoise. L'exagération des récits peut aussi tenir aux pouvoirs surnaturels prêtés aux soldats et incarnés par des figures populaires comme celle de "La Ramée ", étudié par David M. Hopkin ${ }^{39}$. Les représentations de la paysannerie pèsent ainsi sur l'élaboration des récits guerriers et participent à l'édification - souterraine et insaisissable - de cette mémoire populaire de l'épopée impériale dans les campagnes bretonnes. En 1848, Jean-Marie Déguignet, envoyé à l'hospice de Quimper, se souvient ainsi de ces vieux soldats évoquant, entre autres contes et légendes bretonnes, la figure du " grand Napoléon " :

" [...] Parmi toutes ces légendes que ces vieux racontaient, celle de Moscou me paraissait la plus curieuse de toutes. Ils racontaient que là, durant un grand incendie qui dévora la ville entière avec ses habitants, on avait vu l'homme au petit chapeau luttant dans les airs contre un ange audessus de la ville même, et que l'ange finit par précipiter son adversaire dans les flammes. Ca, c'était le signe des malheurs qui lui arrivèrent par la suite $[\ldots]^{40}$."

36. Ces veillées ont été étudiées par Paul Sébillot. Voir Paul SÉBiLLOT, Littérature orale de la Haute Bretagne, Paris, Maisonneuve et Larose, 1882,400 p.

37. Arch. dép. d'Ille-et-Vilaine, Etats civils des communes de Princé et Gahard; 2 R 262 : le maire de Gahard au préfet (14 avril 1853). La propension de l'oncle Toinot, ancien soldat impérial et oncle du narrateur de " la vie d'un simple " à raconté son corps à corps meurtrier avec un soldat russe dans les mariages est, à cet égard, significative. Emile GullLaumin, La Vie d'un simple, Paris, Stock, 1943, première édition 1904, p. 61-62 et p. 96.

38. David. M. HoPKIN, Soldier and Peasant..., op. cit, p. 120.

39. Ibidem, p. 339-346.

40. Jean-Marie DÉGUIGNET, Histoire de ma vie. L'intégrale des mémoires d'un paysan bas breton, édition établie par Laurent Quevilly et Berner Rouz, Ar Releg-Kerhuon, An Here, 
À cette " folklorisation " des récits guerriers il faut ajouter le sens que peuvent donner les collectivités villageoises à l'expérience militaire des vétérans de l'Empire. La spécificité des repères temporels et historiques du monde paysan exclut l'inscription de ces récits dans une chronologie minutieuse de l'Histoire nationale. Ils s'intègrent alors à cette atmosphère de contes et de bavardages, d'histoire familiale et de récits inscrits dans l'histoire locale ${ }^{41}$. Adolphe Orain se souvient ainsi des récits de son grandpère, Jean-Baptiste Delaunay, ancien soldat impérial devenu aubergiste dans la commune de Bain : "Tantôt Papa Delaunay faisait le récit d'une bataille à laquelle il avait assisté. Une autre fois c'étaient des souvenirs de la chouannerie qui étaient invoqués, ou des histoires de chasse, toutes plus extraordinaires les unes que les autres ${ }^{42}$."

Dans ce département touché par les troubles de la guerre civile, l'imaginaire politique et mémoriel des collectivités villageoises s'ordonne également sur les souvenirs de l'épisode révolutionnaire. Les communes "bleues " et patriotes du département peuvent ainsi intégrer à leur manière la parole et l'expérience des vétérans de Napoléon ${ }^{43}$. Cette tendance est remarquable dans la commune "patriote " de Gahard.

Cette commune se distingue en effet par l'engagement armé de sa Garde nationale durant la Révolution et son combat victorieux contre les chouans au mois de novembre 1793. Cet épisode fondateur de la mémoire des bleus de Gahard est entretenu tout au long du XIX ${ }^{\mathrm{e}}$ siècle par la présence au sein du terroir d'un " chêne de la liberté " encore visible en $1914^{44}$. La réactivation de la Garde nationale contre la menace chouanne durant les Cents Jours introduit les vétérans dans le récit patriote gahardais. Ceux-ci rejoignent alors les rangs de cette milice armée et participent, après sa dissolution, à l'agitation d'une nébuleuse patriote dans les premiers temps de la Restauration. La reformation de la Garde nationale dans les communes patriotes au début des années 1830 offre aux vétérans gahardais des postes d'officiers et d'instructeurs, qui font d'eux les fers de lance de cet " esprit bleu ", au moment où s'éteignent les derniers feux de la chouannerie dans

2001, p. 84-85. Il faut toutefois souligner l'aspect singulier de ce témoignage, l'épisode se déroulant en 1848, en pleine période de propagande bonapartiste dans les campagnes bretonnes.

41. Sur les repères temporels du monde paysan, voir Alain CORBIN, Le Monde retrouvé de Louis François Pinagot. Sur les traces d'un inconnu (1798-1876), Paris, Albin Michel, 2002, $1^{\text {re }}$ ed. 1998, p. 179-203.

42. Arch. dép. d'Ille-et-Vilaine, 105 J 3; Fonds Adolphe Orain. Je remercie Jean Le Bihan pour m'avoir signalé ce précieux témoignage.

43. Cette typologie des couleurs politiques locales en Ille-et-Vilaine reprend celle énoncée par Michel LAGRÉE dans Mentalités, Religion et Histoire en Haute-Bretagne au $19^{e ̀ m e}$ siècle : le diocèse de Rennes 1815-1848, Paris, Klincksieck, 1977, 492 p. Dans la commune de Princé, une commune "blanche " et légitimiste, ce sont les anciens chouans qui semblent surtout se distinguer. Voir à ce sujet, Anne Rolland BoulestreAu, Les Notables des Mauges. Communautés rurales et Révolution (1750-1830), Rennes, PUR, 2004, p. 324-337.

44. Jules LuCAS, Un coin d'Ille-et-Vilaine. Gahard, Amicale laïque de Gahard, 1994, p. 103-107. 
le département. Quelques années plus tard, le soutien apporté par la commune au nouveau régime impérial s'inscrit dans un bonapartisme rural largement partagé par les autres communes bleues du département ${ }^{45}$. L'esprit patriote de Gahard trouve alors sa cohérence dans une mémoire qui intègre 1793 , les Cents Jours et la restauration impériale; laissant de fait une place symbolique aux vétérans impériaux dans les cérémonies politiques villageoises sous le second Empire ${ }^{46}$. À Gahard, les anciens soldats de Napoléon sont devenus des " patriotes " avancés. L'imaginaire politique villageois intègre ainsi la parole et l'expérience des anciens soldats leur accordant de fait une marge de reconnaissance au sein de cet esprit patriote.

S'il est impossible de quantifier cette parole, le cadre dans lequel s'opère cette remémoration témoigne donc de la forte imprégnation locale des récits. Ces hommes ne sont pas tous porteurs d'une mémoire à sens unique qui ferait d'eux ces relais du bonapartisme ou ces " professeurs d'énergie nationale " que décrit le mythe du " soldat laboureur 47 ". Leur parole dépend des capacités d'écoute d'un auditoire souvent éloigné des préoccupations nationales et qui, au moins dans ce département, ne semble pas reconnaître - à quelques exceptions près - de notabilité liée à l'expérience militaire. Enfin, l' " enracinement " villageois et l'expérience ordinaire de la conscription napoléonienne pour toute une génération expliquent peut-être la dimension secondaire de l'identification militaire, au moins avant 1858. Avoir fait les campagnes de l'Empire ne condamnait pas ces hommes à faire figure, toute leur vie, d'anciens soldats de Napoléon. Aussi serait-il préférable d'évoquer une identité fuyante, affleurant au grès des circonstances politiques, des sollicitations d'un éventuel auditoire et plus largement de la contingence de parcours toujours singuliers et individuels.

La fragilité de tels processus ne saurait être généralisée à l'ensemble de ce groupe, lequel se caractérise avant tout par une très grande diversité sociale, culturelle, politique et régionale. Ces processus identitaires ont été suivis à l'échelle de collectivités villageoises bretonnes éloignées - sans être cloisonnées - d'une certaine culture nationale et militaire, bien différentes à cet égard des campagnes de l'Isère ou de la Meuse; et dans lesquelles les associations d'anciens combattants sont alors inconnues. Ils constituent néanmoins une alternative aux représentations envahissantes du vieux briscard bonapartiste mises en scène dans les défilés des médaillés de

45. Yann Lagadec, Pouvoir et Politique en Haute Bretagne rurale..., op. cit. p. 610.

46. Les vétérans de Gahard sont mis à l'honneur lors de la fête du 15 août. Arch. dép. d'Ille-et-Vilaine, 1 M 186 : rapports des maires sur les festivités du 15 août.

47. Ces représentations sont alors davantage partagées par les élites que par les couches populaires auxquelles appartiennent une majorité de vétérans. Voir à ce sujet les réflexions de David. M. Hopkin, Soldier and Peasant..., op. cit., p. 285. Sur le mythe du " soldat-laboureur " voir Gérard de PUYMÈGE, Chauvin, le soldat-laboureur. Contribution à l'étude des nationalismes, Paris, Gallimard, "Bibliothèque des Histoires ", 1993, 293 p. 